\title{
APRENDIZAJE SIGNIFICATIVO EN ESTUDIANTES DE AGRONOMÍA
}

\section{MEANINGFUL LEARNING BY AGRONOMIE-STUDENTES}

Referencia del artículo

Guevara, N. S. (2022). Aprendizaje significativo en estudiantes de agronomía. Revista Científica del Sistema de Estudios de Postgrado de la Universidad de San Carlos de Guatemala, 5(1), 9-14. DOI: https://doi.org/10.36958/sep.v5i1.91

\author{
Nery Saúl Guevara \\ nery.guevara@ues.edu.sv \\ https: / / orcid.org/0000-0002-9528-5090 \\ Doctorado en Educación con especialidad en Educación Superior \\ Universidad de El Salvador
}

Fecha de recibido: 15/10/2021

\section{RESUMEN}

OBJETIVO: potenciar el aprendizaje significativo en los estudiantes de Sociología rural de primer año de agronomía, situada en una línea de investigación de aprendizaje, educación y desarrollo de la educación superior. MÉTODO: fueron necesarios métodos teóricos y empíricos sustentados en el método dialéctico-materialista, que es un método general en que confluyen las ciencias, integra el análisis del comportamiento cuantitativo y cualitativo del objeto de investigación. La población objeto de estudio fueron 40 estudiantes del curso Sociología rural. RESULTADOS: el $50 \%$ de los estudiantes encuestados (20), manifiestan que para aprender lo hacen por memorización. CONCLUSIÓN: los planteamientos teóricos propuestos y los resultados parciales de carácter cuantitativo y cualitativo, para conocer el aprendizaje y la calidad de la práctica profesional, sugieren cambios en los procesos de enseñanza aprendizaje para potenciar el aprendizaje significativo que estimulen el desempeño y satisfacción laboral en beneficio propio y de la sociedad en general.

\section{PALABRAS CLAVE}

Aprendizaje, enseñanza, aprendizaje significativo, estudiantes
Fecha de aceptado: 05/01/2022

\section{ABSTRACT}

OBJECTIVE: to promote meaningful learning in rural sociology students in the first year of agronomy, situated in a line of research on learning, education and development of higher education. METHOD: theoretical and empirical methods based on the dialectical-materialist method were necessary, which is a general method in which the sciences converge, integrating the analysis of the quantitative and qualitative behavior of the research object. The population under study were 40 students from the Rural Sociology course. RESULTS: $50 \%$ of the students surveyed (20) state that to learn they do so by memorization. CONCLUSION: the theoretical approaches proposed and the partial results of a quantitative and qualitative nature, to know the learning and the quality of professional practice, suggest changes in the teaching-learning processes to promote meaningful learning that stimulate performance and job satisfaction for the benefit own and society in general.

\section{KEYWORDS}

Learning, teaching, meaningful learning, students 


\section{INTRODUCCIÓN}

El principio para potenciar el aprendizaje significativo en los estudiantes, supone comienza con los conceptos. "Porque sin conceptos no hay comprensión, no hay desarrollo cognitivo. El hombre vive en un mundo de conceptos. Sin embargo, incomprensiblemente, la importancia de los conceptos es ignorada en la escuela, en especial en la educación científica" (Moreira, 2008).

Este trabajo, tiene como objetivo potenciar el aprendizaje significativo en los estudiantes de Sociología rural de primer año de Agronomía. En ello se abordan esencialmente el concepto del aprendizaje significativo integrados a otro gran número de conceptos que deben aprenderse $y$, en el que son identificados en los procesos de enseñanza-aprendizaje por los sujetos docentes-estudiantes, en la búsqueda de satisfacer las necesidades y expectativas como exigencia para la mejora institucional y de la sociedad.

Contiene un resumen, en el que se hace una breve descripción de las ideas fundamentales del artículo y las palabras claves. De igual modo, el cuerpo del artículo, contiene los planteamientos teóricos de los autores que refieren el aprendizaje significativo, la metodología empleada en el proceso enseñanza aprendizaje. Así mismo, se presentan algunos resultados que arrojó una prueba piloto en la aplicación de los instrumentos que genera el estado del aprendizaje significativo.

Para ello, se propone resolver los planteamientos teóricos siguientes: el proceso de enseñanza aprendizaje universitario, el aprendizaje significativo, el rol del profesor $y$ el estudiante en el aprendizaje significativo. Seguidamente, se describen las conclusiones, en las cuales se explica que este escrito está fundamentado en el capítulo uno y dos de la tesis del Doctorado en Educación, con Especialidad en Educación Superior de la Facultad Multidisciplinaria de Occidente de la Universidad de El Salvador y, finalmente, las referencias que lo sustentan.

\section{MATERIALES Y MÉTODOS}

En la metodología se consideró el método dialéctico-materialista, para el análisis del comportamiento del objeto de investigación con alcance descriptivo. Entre los métodos empíricos y técnicas utilizadas en la investigación está la encuesta y la entrevista. En la fase exploratoria, se contó con una muestra de 40 estudiantes encuestados y al azar entre ellos se seleccionaron a 10 estudiantes para la realización de la entrevista respectivamente.

\section{RESULTADOS}

Para reflejar resultados, este autor desarrolla una explicación teórica acerca del aprendizaje significativo como proceso enseñanza aprendizaje, y a nivel empírico desarrolla el estado actual, para luego sistematizarlo y evaluarlo y tomar decisiones. Para fundamentar el aprendizaje significativo, se hizo necesario el desarrolló de los planteamientos siguientes:

\section{El proceso de enseñanza aprendizaje universitario}

La formación del estudiante y la práctica educativa del docente universitario en un contexto de cambio, no puede continuar con exposiciones en que el maestro es el centro en el proceso enseñanza aprendizaje se dedican a transmitir conocimientos acríticos, memorísticos o mecanizados, haciendo del 
estudiante un ente pasivo, relegado de toda participación en las decisiones propias que le competen.

A juicio de Caira, Urdaneta, \& Mata (2014), enfatizan que "Desde una perspectiva constructivista, el papel del docente se orienta bajo la figura de mediador y propiciador de situaciones de aprendizaje, en las que el alumno es considerado como participante activo del proceso".

En efecto, Carranza (2017), plantea que una de las exigencias actuales para mediar en el proceso enseñanza aprendizaje a nivel universitario es la incorporación de las tecnologías en el aula, la capacitación de los docentes, en el uso de estas herramientas con fines pedagógicos, al mismo tiempo en que su esfuerzo se vea enfocado en lograr que los estudiantes sean eficaces, autónomos y que practiquen de la mejor manera la autogestión.

Para el autor, al docente y a la institución le corresponde asumir compromisos para emprender acciones que les permitan transitar de la formación tradicional y/o combinar con la mediación tecnológica como estrategia pedagógica para aprovechar los beneficios que se generen de los procesos de enseñanza aprendizaje para el estudiante

\section{El aprendizaje significativo}

Evidentemente, a la escuela actual y comunidad educativa se le plantean desencuentros con el paradigma constructivista, constituidas por una serie de situaciones problemáticas que ocurren en el aula, en el que predominan esquemas y conceptos que no posibilitan construir significados por parte del alumno, ya sea porque se vuelve monótono, desmotivado que no le permite visualizar el verdadero significado de los conceptos.
Uno de los componentes más importantes implícitos en el proceso de enseñanza-aprendizaje es el aprendizaje significativo, lo que según el autor supone ayudas al docente para relacionar y argumentar los contenidos que se desarrollen en el aula.

De acuerdo con Ausubel (1983), citado por Moreira (2017), da a conocer que la teoría del aprendizaje significativo, ofrece un marco apropiado para el desarrollo de la labor educativa, que se caracteriza por una interacción docente-estudiante, entre los conocimientos más relevantes que posee el aprendiz y las nuevas informaciones a adquirir, de tal modo que surjan significados producidos a partir de la relación que se establece con la estructura cognitiva del aprendiz.

Para el autor, el estudiante al entrar en contacto con el objeto de estudio, lo asimila mediante la interacción relacionándolo con sus experiencias, que está inmerso en un entorno sociocultural, adquiere nueva información y lo enlaza con el conocimiento previo, internalizándolo en la estructura mental que lo procesa para producir nuevos conocimientos.

\section{El rol del profesor y el estudiante en el aprendizaje significativo}

En las últimas décadas se ha cuestionado el rol tradicional del profesorado. "Al personal docente se le exige la dirección científica del proceso pedagógico. Sin embargo, la práctica de la educación refleja algún nivel de improvisación del docente y un ajuste a las particularidades de los sujetos" (Ortíz, 2013).

Para el autor, la práctica pedagógica que incurre en el desempeño docente, se basa en exposiciones magistrales, sin que el sujeto haya interactuado con el profesor y el contenido a aprender, se le ha impregnado de 
conceptos sin el respectivo sentido y significado, enseñando espontáneamente al alumno lo cual es afectado en su formación.

Importa, y por muchas razones, el estudiante en su proceso de enseñanza-aprendizaje encuentra que la clase no le es correspondida en su interés y necesidad, pierde el sentido de su atención en el tema que se desarrolla.

Según Fernández (2018), alude que el rol del docente es clave porque hay que mediar estos problemas y al mismo tiempo lograr que los jóvenes no se distraigan, ya que pierden el interés rápidamente si sienten que la clase puede llegar a ser, según lo que ellos consideran, monótona o aburrida.

Para el autor, el sostener el acto educativo por parte del profesor implica motivar, comprender y movilizar el sentir del estudiante, organizando actividades que respondan a las necesidades e intereses, que faciliten el aprendizaje.
Por consiguiente, Guerra, Rodríguez, \& Josué (2019), consideran que la misión última del profesorado debe ser el de favorecer la reflexión, la capacidad de pensar y la toma de confianza en el alumnado. Este nuevo modelo requiere que los/las docentes se actualicen en la formación de las nuevas metodologías educativas y sobre todo lo pongan en práctica.

Para el autor, estas prácticas innovadoras son incipiente en los sistemas escolares por el que existen en su mayor parte metodologías basadas en la enseñanza tradicional, los estudiantes asumen un rol pasivo negándosele por sí solo a ser activo protagónico en el aula. En muchos casos, las tareas que los alumnos tienden a desarrollar, carecen de contenidos que no se relaciona con la realidad de su entorno educativo.

\section{Tabla 1. Resultados de la encuesta}

\begin{tabular}{lcc}
\hline \multicolumn{1}{c}{ Preguntas realizadas en la encuesta. } & Indicadores & $\%$ \\
\hline 1. ¿El material empleado en el proceso & Muy atractivo & 10 \\
\cline { 2 - 3 } enseñanza-aprendizaje, le ha sido atractivo & Bastante atractivo & 20 \\
\cline { 2 - 3 } en el logro del aprendizaje? & Poco atractivo & 60 \\
\cline { 2 - 3 } & Nada atractivo & 10 \\
\hline 2. En el desarrollo del tema por el docente, & Repetición & 30 \\
\cline { 2 - 3 } ¿Cómo adquiere conocimientos el & Memorización & 50 \\
\cline { 2 - 3 } estudiante? & Significativo & 20 \\
\hline 3. ¿Interactúa usted con los docentes para & Bastante & 10 \\
\cline { 2 - 3 } decidir los contenidos del programa a & Poco & 60 \\
\cline { 2 - 3 } aprender? & Nada & 30 \\
\hline 4. ¿Cuál es el papel que desempeña el & Pasivo & 60 \\
\cline { 2 - 3 } estudiante en el proceso enseñanza- & Reflexivo & 30 \\
\cline { 2 - 3 } aprendizaje? & Crítico & 10 \\
\hline
\end{tabular}

Fuente: Guevara, 2021, Información de base de datos del estudio de campo 
El material empleado en el proceso enseñanza aprendizaje para el logro del aprendizaje, el $60 \%$ (24) de los estudiantes expresan que son poco atractivos, el $20 \%$ (8) lo consideran bastante atractivos, el 10\% (4) muy atractivo e igual, el $10 \%$ (4) lo consideran nada atractivo; por otra parte que, el $50 \%$ de los estudiantes (20) manifiestan que para aprender lo hacen por memorización, el 30\% (12) lo hacen por repetición y el 20\% (8) lo aprenden por aprendizaje significativo.

De los 40 estudiantes encuestados, el 60\% (24), manifestó que es poca la interacción que tiene con el docente, el 30\% (12) no interactúa y, el $10 \%$ (4) de los estudiantes interactúan bastante; asimismo, del papel que desempeña el estudiante en el proceso enseñanza aprendizaje, el $60 \%$ (24), expresan que su papel es pasivo; el 30\% (12) reflexivo y el 10\% (4) desempeña su papel como estudiante crítico.

En la entrevista realizada a 10 estudiantes sobre las tareas que les deja el profesor, son involucrados en su propio aprendizaje, los estudiantes expresan que solo si los involucran, caso contrario, hay estudiantes que lo hace solo por salir del compromiso. En cuanto al material didáctico que se emplea en el proceso enseñanza aprendizaje para facilitar el aprendizaje significativo, los estudiantes manifiestan que estos deben se atractivos, claros y concisos.

\section{DISCUSIÓN}

Teniendo en cuenta que los estudiantes en su proceso de aprendizaje deben ser constructores de conocimientos, la encuesta refleja que, en el aula, la mayoría de estudiantes (24) se muestran pasivos en el proceso de enseñanza aprendizaje, lo que a juicio de Caira, Urdaneta, \& Mata (2014), señalan que "Desde una perspectiva constructivista, el papel del docente se orienta bajo la figura de mediador y propiciador de situaciones de aprendizaje, en las que el alumno es considerado como participante activo del proceso".

Tal como lo hace notar Ausubel (1983), citado por Moreira ( 2017), refiere que la teoría del aprendizaje significativo, ofrece un marco apropiado para el desarrollo de la labor educativa, que se caracteriza por una interacción docente-estudiante, entre los conocimientos más relevantes que posee el aprendiz y las nuevas informaciones a adquirir, de tal modo que surjan significados producidos a partir de la relación que se establece con la estructura cognitiva del estudiante. Lo que el autor, supone que la actividad más importante que ha de realizarse en el sistema escolar es el proceso de aprendizaje significativo.

El material didáctico que se emplea en el proceso de enseñanza aprendizaje, puede ser o no atractivo para el estudiante para facilitar o no el aprendizaje significativo, de acuerdo con Fernández (2018), el rol del docente es clave porque hay que mediar estos problemas, y al mismo tiempo lograr que los jóvenes no se distraigan ya que pierden el interés rápidamente si sienten que la clase puede llegar a ser, según lo que ellos consideran, monótona o aburrida.

\section{REFERENCIAS}

Ausubel, D., Novak, J., \& Hanesian, H. (1983). Psicología educativa: un punto de vista cognoscitivo - David Paul Ausubel, Joseph Donald Novak, Helen Hanesian Google Libros

Caira, J., Urdaneta, E., \& Mata, L. (2014). Redalyc. Estrategias para el aprendizaje significativo de procesos de fabricación mediante orientación constructivista

Carranza, M. D. (2017). Enseñanza y aprendizaje significativo en una modalidad 
mixta: percepciones de docentes y estudiantes. Rev. Iberoam. Investg. Desarro.Edu.doi: https://doi.org/10.23913/ ride.v8i15. 326

Fernández, R. (2018) ¿Por qué el constructivismo es el método de enseñanza/aprendizaje de la nueva generación? Catálogo Digital de Publicaciones DC (palermo.edu)

Guerra, M., Rodríguez, J., \& Josué, A. (2019). Aprendizaje colaborativo: experiencias innovadoras en el alumnado universitario. Revista de estudios y experiencias en educación. DOI:10.21703/rexe. 20191836guerra5

Moreira, M. (2008). Conceptos de la educación científica: ignorados y subestimados (ull.es)

Moreira, M. (2017). Aprendizaje significativo como un referente para la organización de la enseñanza. Archivos de ciencias de la educación. https://doi. org/10.24215/23468866e029

Ortiz, A. (2013).(PDF) Modelos Pedagógicos y Teorías del Aprendizaje (researchgate. net)

\section{SOBRE EL AUTOR}

\section{Nery Saúl Guevara}

Tiene 26 años de ejercer la docencia en la Universidad de El Salvador; Ingeniero Agrónomo, Lic. en Ciencias Políticas, Maestría en Métodos y Técnicas de Investigación. Actualmente cursando el Doctorado en Educación Superior de la Universidad de El Salvador.

Conflicto de intereses

Declara no tener ningún conflicto de intereses.

Declaración de consentimiento informado

El estudio se realizó, respetando el Código de ética y buenas prácticas editoriales de publicación.

Derechos de uso

Copyright@ 2022 por Nery Saúl Guervara

Este texto está protegido por una licencia Creative Commons 4.0. Internacional

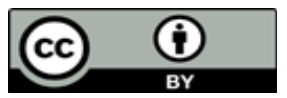

Usted es libre para compartir, copiar y redistribuir el material en cualquier medio o formato y adaptar el documento, remezclar, transformar y crear a partir del material para cualquier propósito, incluso comercialmente, siempre que cumpla la condición de atribución: usted debe reconocer el crédito de una obra de manera adecuada, proporcionar un enlace a la licencia, e indicar si se han realizado cambios. Puede hacerlo en cualquier forma razonable, pero no de forma tal que sugiera que tiene el apoyo del licenciante o lo recibe por el uso que hace. 